\title{
An Integrated robotic system for antipersonnel mines detection
}

\author{
Eric Colon ${ }^{\mathrm{a} *}$, Ping Hong ${ }^{\mathrm{a}}$, Jean-Claude Habumuremyi ${ }^{\mathrm{a}}$, Ioan Doroftei ${ }^{\mathrm{a}}$, \\ Yvan Baudoin ${ }^{\mathrm{a}}$, Hichem Shali ${ }^{\mathrm{b}}$, Dragomir Milojevic ${ }^{\mathrm{c}}$, Jérôme Weemaels ${ }^{\mathrm{d}}$ \\ ${ }^{a}$ Robotics Centre, Royal Military Academy, Avenue de la Renaissance 30, B-1000 Brussels Belgium \\ b Department of Electronics and Information Processing VUB-ETRO-IRIS, Vrije Universiteit Brussel, \\ Pleinlaan 2, B-1050 Brussels \\ ${ }^{\mathrm{c}}$ Service des Systèmes Logiques et Numériques, Université Libre de Bruxelles 50, Av. F.Roosevelt, CP \\ 165/57 B-1050 Bruxelles \\ ${ }^{d}$ SCMeRO, Université Libre de Bruxelles 50, Av. F.Roosevelt, CP 165/42 B-1050 Bruxelles \\ phone: +3227376552 \\ fax: +3227376547 \\ eric.colon@mapp.rma.ac.be
}

Abstract: In this paper robotic systems for antipersonnel mines detection are presented. Robots with different locomotion principles have been developed to carry mines detection sensors. In this project, many different aspects including mechanics, control and positioning have been addressed. After a short review of the project and a description of the system components, the control and communication architecture of the robotic detection systems is presented. One of the most important issues in this application was the synchronisation of the processes between the microcontrollers and remote computers. The method that has been implemented has proven to be robust and the different systems have been used to acquire very useful data for the research community.

Keywords: robotics, remote control, microcontroller, human machine interface, sensors, visual servoing.

\section{Introduction}

The terrible antipersonnel landmines plague represents a real challenge for the research community. Antipersonnel mines kill or mutilate tens of people every day. Humanitarian deminers still use classical manual methods because heavy demining vehicles cannot achieve a satisfying destruction percentage. This work is very slow, tedious, dangerous and costly. Furthermore, the detection is not always reliable. Improvements can be made by developing new sensors, by automating the detection sequence and by using different sensors simultaneously (Baudoin \& Colon, 1999). The Royal Military Academy, leading the Belgian 
project Hudem, is focusing on the development of new data processing and fusion algorithms (Milisavljevic 1999), on improvement of Ground Penetration Radar (GPR) (Scheers 1999) and on robotic systems that carry mines detection sensors.

Among the different ways robots could help human deminers, the scenarios described below are the most realistic. Small autonomous vehicles equipped with different sensors could run around an area to delimit the surface that is really polluted with mines. This phase when done manually is the most dangerous one because deminers are working faster and are taking more risks than during systematic detection. To study this first aspect, a small wheeled electrical vehicle named Tridem has been developed (figure 1). A first indoor prototype was build to demonstrate the concept feasibility (Alexandre, Weemaels, Doroftei \& Colon 1998). A second version with more powerful motors and a modified frame has been developed for outdoor trials.

Once the actual mined area is delimited, a systematic scanning process can begin. It has been proved that the use of different sensors could drastically improve the detection efficiency and reliability. However, the data fusion process requires the registration of the data acquired by the different sensors. This requirement justifies the work done on the two other systems: the first one which is a sliding pneumatic robots with a 2 degrees of freedom scanner (figure 2) and the second one which is a modified Explosive Ordnance Disposal (EOD) vehicle that carries a three-dimensional Cartesian scanning system (figure 3) (Colon, Baudoin \& Alexandre 1998). The fact that the three systems have very different robotic architectures introduced some additional challenges in the development of the control and interface architecture. Also the different sensors used in the project and more particularly their interface influenced the system implementation.

The last aspect considered in this project is the determination of the robot's location in the field. This is required for navigation but also for automatic production of detection maps. For this purpose, a visual servoing system based on a pan-and-tilt colour camera has been developed. (Hong, Sahli \& Baudoin 1998). This system tracks a colour beacon mounted on the robot and sends in real-time the three-dimensional position of the sensor to the main control computer. The principle is shown in figure 4 .

Besides mobility trials, the systems have been used on dummy domestic minefields to record data for the searchers working on sensor development and data fusion algorithms.

This article begins with an overview of the different components of the robotic acquisition systems. Next, the control and communication architecture is described. The emphasis is put on the control of the scanning and 
on the synchronisation between the distributed processes. Finally some results are presented and future work is discussed.

\section{System overview}

Basically, the scanning robotic systems are composed of the following elements:

- The vehicles with their possible scanning device,

- The mine detection sensors,

- The tracking and location system.

As described in the preceding section the robotic systems can be divided into two categories: the ones having a scanning device that can be equipped with different sensors and the wheeled robot that simply carries a single sensor. In this case, the scanning of an area can be obtained by moving the robot body itself.

The mechanical systems need to be controlled and their motion must be synchronised with the data acquisition. The vehicle motion is also synchronised with the tracking and location process.

The vehicles and the scanners are described in this section; the control and communication architecture is presented in section 3 .

The first vehicle available in the project is called Hunter (figure 3); it is a small caterpillar vehicle that was used by the Belgian Army for anti-terrorism operations. The original manual control system of the vehicle has been interfaced with a microcontroller such that the vehicle can now be controlled with a computer. The scanner is actually a Cartesian robot with three degrees of freedom. A DC servomotor coupled with a planetary gear-head actuates each axis. An optical digital encoder is used for position and speed feedback and contact switches have also been placed at the end of each axis. The useful area is 850 by $500 \mathrm{~mm}$ and the vertical axis has a travel distance of $500 \mathrm{~mm}$. The system can be used on both sides of the vehicle and is powered with batteries or with a power supply.

The second robot (AMRU4) is a pneumatically actuated walking machine (figure 2). Two sliding frames allow a linear motion and a rotating cylinder is used for changing the motion direction. The upper part of the robot is a two degrees of freedom scanner that can carry different kinds of mine detection sensors. As mentioned in the introduction, to develop efficient detection methods, precise and reliable data are essential. This fact justifies the development of those two systems, which are able to acquire multi-sensor data. 
The third vehicle called Tridem (figure 1) has a triangular shape and stands on three wheels. The wheels are connected to the frame as a three arms star. The frame supports the electronics and the batteries. The robot is equipped with three driving and three steering motors. The power is transmitted to the driving motors by copper brushes. This solution gives the wheels a complete rotation freedom around the steering axis. System modularity was one of the basic requirements for this robot. The wheels can be removed and replaced very easily because all the wheels are identical and are fastened with fast screw connections. The electrical connections are also very easy; the wires (control signal and power) are connected through standard DB9 connectors which are embedded into the frame. It is an omnidirectional vehicle that has been designed for highly unstructured grounds. This platform could be used to detect mines in areas which are dangerous for human deminers.

\section{Control Architecture}

\subsection{The components of the control architecture}

The whole system has a multi-processing architecture and comprises the following components (figure 5):

- The HMI computer,

- An embedded computer for data acquisition and communication with the HMI computer,

- The motion controller (microcontroller),

- The visual tracking and location computer.

The following function are distributed among the different processing systems:

- Motion control

- Processes Synchronisation

- Data Acquisition

In this application, two kinds of interfaces are needed: communication interfaces (serial, Ethernet) and acquisition interfaces (serial, GPIB, frame grabber).

The subsequent subsections give and overview of the different components of the control architecture. The last subsection describes the integration and synchronisation of the different systems and processes. 


\subsection{The Master PC}

The control architecture comprises two loops: a high level control loop that runs on a PC and synchronises high level processes and a low level control loop running on a microcontroller which performs common control functions (position and speed control). The Master PC is responsible for the synchronisation of the robot motion with the positioning system and for the synchronisation of the scanning with the data acquisition. It sends configuration and trigger commands to the microcontroller trough a serial link and performs the data acquisition.

The role of the Master PC can be played by the HMI PC, when it is directly connected to the vehicles and to the sensors, or by the embedded PC which communicates via a TCP/IP link with the HMI PC. This remote acquisition can for the moment only be performed with the metal detector (RS232 interface). The direct connection is required when acquiring data from the GPR because it is an instrumentation system that cannot be easily displaced. It uses a normal PC HPIB interface card connected to a scope for acquiring data. When a portable sensor is available, it will be possible to easily integrate it into the mobile system.

\subsection{The motion and scanning controller}

The three robotic systems use the same basic electronics; the low-level control is realised with a 32-bit microcontroller that communicates through a serial link with the Master PC. They can be remotely controlled through the same HMI, a program called CoRoDe (Control of Robots for Demining). The HMI manages all control aspects: vehicle and scanning control, communication and data acquisition. A high interactivity and user-friendliness is reached through the use of efficient programming techniques (see section 3.7).

The 32-bit microcontroller is very versatile and powerful; it performs different functions depending on the system:

- It controls electrical motors (scanner, Tridem),

- It monitors signals coming from contact switches,

- It generates commands (replacing a PLC) for controlling the valves on the pneumatic robot,

- It generates analogue signals for the tracks of the caterpillar vehicle,

- It hosts the main communication loop with the Master PC.

The specific characteristics of the control methods are given in the remainder of this subsection. 
What concerns the Hunter, two control levels can be distinguished: a software only loop that manages communication through the serial interface and the motion control functions which are interrupt-based. The serial port is polled and a switch-case instruction fires the function corresponding to the received code. Main high level control functions for the Hunter are: set scanning limits, set position, set speeds, move to area limits, start scanning ... During the scanning, synchronisation signals are sent to the Master PC to trigger the data acquisition. Two scanning modes are available: a continuous mode and a discrete mode. In the discrete mode, the scanner stops at every step waiting for the end of the data acquisition. The acquisition step can be changed in the set-up dialog box of the HMI.

In the moveTo function, the desired goal co-ordinates are copied into shared variables that are read by the motion control functions. These functions are called by interrupts at fixed intervals (a $4 \mathrm{~ms}$ control period is used). The PI controller controls the motor speed by using the Time Processing Units (TPU) of the microcontroller which are configured to generate PWM signals. A cascade negative feedback control combining two loops has been implemented: the first loop corresponds to a speed negative feedback control (the inner loop) while the other one is for position negative feedback control (the outer loop).

The microcontroller also generates analogue signals to drive the tracks of the Hunter. Two TPU's channels produce two independent PWM signals for right and left tracks. The PWM signals are filtered to generate continuous bipolar analog signasl compatible with the old interface. As the driving motors do not have encoders it actually acts as an open loop controller.

In the case of the walking robot, the microcontroller is essentially used as a software PLC. The control is implemented like a finite state machine and all outputs are binary signals. The TPU's are used to read the position signal from incremental linear sensors placed along the pneumatic cylinders. The main loop manages the communication with the master PC.

The control of the Tridem is similar to the one of the Hunter but requires all TPU's of the controller. The TPU's generate six PWM signals and capture the digital encoders' signals from the steering motors. The speed of the driving wheels are measured with tacho-generators whose signals are converted by the internal AD converters. A classical PI algorithm is used for controlling the wheels.

Different configurations are possible (figures $6 \mathrm{a}, 6 \mathrm{~b}$ and $6 \mathrm{c}$ respectively):

- Translate: all wheels are parallel, 
- Spin: the wheels are tangent to a circle,

- Free: the normal motion mode in which the three wheels are separately commanded to follow arbitrary trajectories.

This later mode is preferred for the detection because it keeps the metal detector pointed along the motion direction.

\subsection{Sensors and acquisition interfaces}

Three different sensors have been successfully used in the project: a metal detector (MD), a Ground Penetration Radar (GPR) and an infrared camera. A fourth one, a pyrometer, has been tested but abandoned due to unsatisfactory results. The data acquisition process requires different interfaces: the metal detector has a serial interface, the GPR data are read through the GPIB interface of a high speed oscilloscope, the images coming from the infrared camera are captured with a frame grabber (through a cable or a wireless connection). We will see in the subsection 3.7 how the sensors' characteristics influence the scanning process and the way the control is realised.

\subsection{Communication}

A serial communication allows the transmission of commands between the Master PC and the microcontroller (the transmission speed is 9600 baud). Radio Ethernet links (protocol 802.11) are used to communicate between the HMI PC and the embedded PC.

\subsection{Location and tracking}

A location system determines the robot's positions which are used to automatically generate a map of the detections (Hong 2001). A pan-and-tilt colour camera tracks a coloured target mounted on the vehicle. Every frame the ball object is extracted from the image (figure 7) and an estimation of its position is computed. As the robot moves, the camera follows the target in order to keep it in the centre of the image. Furthermore, the size of the target is kept constant by using a motorised zoom and the value of the zoom gives an estimation of the distance. As the orientation of the camera is also known, the ball is completely located in the field. In favourable conditions (uniform background, constant illumination) a precision of $30 \mathrm{~cm}$ at distances up to 15 
$m$ is reached, which is enough for this application. In order to increase the precision of the location process in varying conditions the coupling of the camera with a laser telemeter is now considered.

\subsection{Human Machine Interface}

The graphical user interface of the control program CoRoDe is shown in figure 8 . This program offers the following functions:

- Control of the vehicle,

- Configuration and control of the scanning system,

- Configuration of the sensors,

- Data visualisation,

- Data archiving,

- Mapping.

The main area is devoted to data visualisation. It can alternatively display a map with successive locations of the robot or the sensors' data.

Displayed data can result from two different acquisition methods:

- Global acquisition:

- Infra-red images

- Video images (used to view and record pictures from the scanned area)

- Sequential acquisition:

- Single values organised as a $2 \mathrm{D}$ image (MD)

- One-dimensional data merged into a 3D volume (GPR).

The map contains a reference frame and consecutive positions of the robot and scanned areas. The user can switch between either configuration by clicking on a button in the toolbar.

Data acquired during the scan process are saved in two different formats: first as binary data for later processing (double for GPR, double word for MD) and as 8 bits grey scale raw images for direct visualisation. The data acquisition, scanning, location computation and vehicle motion are integrated into a sequence that is controlled by the user with button commands lying in a single toolbar. The interface is simple and intuitive thanks to the use of well-known symbols (VCR-like) and standard colours (see figure 8). In this application, it 
was a requirement to let the user keep the control of the process; at every moment the user can pause, resume or stop the operations.

It is also essential to provide information during internal processing or timeouts. In this case, sensors' data are drawn on the screen as the scanning progresses. The position of the scanner relative to the maximum positions, the status of the scanning sequence and the main options are also presented to the user and regularly updated. Finally the use of additional communication threads (see next section) preserves the interaction with the user interface. The next section provides implementation details about the communication and the synchronisation of processes.

\subsection{Control Process, communication and synchronisation}

The robotic systems used for sequential data acquisition have a more complex control architecture than the wheeled robot. The division of the control between the microcontroller and the Master PC requires a permanent synchronisation of the processes. The main program runs on a computer under the Windows operating system (OS) (Windows98 was used because at that time it was the only windows version compatible with the force-feedback joystick drivers, now Windows2000 or XP could be used as well). The communication with the microcontroller runs in its own thread in order to keep the interactivity of the user interface. Each event coming from the microcontroller (begin or end of line, position trigger for data acquisition) through the serial port is intercepted by the communication thread. For each event, corresponding messages are posted to the view window (the program is implemented with the MFC document-view scheme). Each message results in a call to the corresponding function of the window class (examples of such messages are acquiredata, savedata,...). When using the discrete scanning mode, this process is synchronous and the microcontroller blocks until it receives the acknowledgement of the main process. The consequence is that the scanning is not continuous but stops at every step (typically every $2 \mathrm{~cm}$ ). This was required by the metal detector because when triggered, this sensor has an acquisition latency of about $200 \mathrm{~ms}$ which corresponds to $2 \mathrm{~cm}$ for a speed of $10 \mathrm{~cm} / \mathrm{s}$ and consequently to a $4 \mathrm{~cm}$ shift between the forward and

backward scanning lines. This synchronisation also removes the need to take into account the delays introduced by the OS message queuing mechanism and the acquisition duration. The communication thread 
then enters in a blocking section and waits until the data acquisition has been performed and the lock released to send the acknowledge message to the microcontroller.

Realising the communication between two threads by messages allows to completely uncouple them (the other way is to pass objects' pointers to the control thread and to directly call functions from it). At each step, the position of the scanner in the user interface is updated, data is acquired, plotted and saved.

However, this asynchronous control has two drawbacks: the scanning process is slower and it can produce vibrations due to resonance phenomena. As mentioned earlier, the first limitation is also due to the sensors and cannot be avoided without changing the sensors themselves. Introducing random delays to avoid resonance frequencies can eliminate the second problem. Other possibilities for dealing with the acquisition latency is to calibrate the delays and to shift the data every two lines at the end of the acquisition or to always scan in the same direction. The first solution was rejected by the data processing team because it introduces some extra pre-processing and can alter the data. The second solution has as consequence to double the scanning duration and to cancel the benefit of the continuous scanning; it was consequently not considered.

What concerns the Tridem, there is no such synchronisation between the robot and the Master PC because the communication process is essentially asynchronous. The microcontroller receives configuration or motion commands and executes them. An acknowledgement is sent after the reception of the command, the execution is started and a new command is read from the serial port.

\section{Results and future work}

The two systems equipped with scanners have been successfully used to test the GPR prototype and to acquire registered multi-sensors data (figure 9). These data have been very valuable for the searchers working on fusion methods. The wheeled robot has also been demonstrated as a remotely controlled mine detection system.

Different elements have contributed to the success of this project:

- The use of the same microcontroller for different robots,

- The use of object-oriented programming techniques and language that allow to easily reuse building blocks in developing new applications or modifying existing ones,

- The hierarchical model of the control, 
- The user-friendly user interface.

But despite these benefits, the application presents several limitations:

- It is a monolithic application where control and interface cannot be easily separated,

- It is OS specific,

- Adding a robot with new capabilities or a sensor with new display requirements would demand important modifications of the program.

These drawbacks are not unique for this application but are common characteristics of classic programs.

New ways for implementing the control and visualisation software are consequently considered: components and distributed components like CORBA or Java RMI are possible candidates. The requirements for the new architecture are listed below. It should:

- Provide an universal User Interface,

- Have Plug \& Use capability,

- Be open to existing and future robots,

- Allow the concurrent control of different robots,

- Allow the easy reconfiguration of the application (addition or removal of components: robots, sensors, users),

- Allow several users to collaborate,

- Allow the users to incorporate their own control algorithms.

\section{Conclusion}

This paper has presented the results achieved by the Robotic Workgroup within the Belgian funded project Hudem. Robots using different locomotion techniques have been successfully used to acquire sensor data and to test and validate utilisation methods in different scenarios. In this paper, the control and programming architectures of these systems have been described. The use of object oriented techniques for application development and the reuse of the same control hardware have contributed to the success of this project. However, in the next future new programming techniques will allow developers to write more open and reusable applications. 
At this stage, it has not been proven that robotic detection of mines works better or faster than human deminers. But the obtained results are encouraging and pave the way for an integrated solution that will some day help to solve this terrible plague.

\section{Acknowledgements}

This project would not have been possible without the contribution of all the searchers involved in the Hudem project and without the funding by the Belgian Ministry of Defence and the Secretary for Cooperation and Development.

\section{References}

Alexandre P., Weemaaels J., Doroftei I. \& Colon E., (1998) Development of a high mobility wheeled robot for humanitarian mine clearance, Proc. Robotic and semi-robotic ground vehicle technology, Aerosense SPIE, Orlando, USA.

Baudoin Y. \& Colon E. (1999) Humanitarian Demining and Robotics: a difficult challenge, Proc. Clawar99, Portsmouth, UK.

Colon E., Baudoin Y., Alexandre P. (1998) Development of mobile robots for mines detection, Proc. Mechatronics 98 , Skovde, Sweden.

Hong P., Sahli H. \& Baudoin Y.(1998) Color Target Detection and Tracking, Proc. Clawar '98, Brussels, Belgium

Hong P. (2001) Visual servoing for robot navigation: application in humanitarian demining, Ph. D. Thesis, Free University of Brussels, Belgium.

Milisavljevic N. (1999) Mine shape detection and data fusion considerations, Proc. Hudem Symposium, RMA, Brussels, Belgium.

Scheers B. (1999), Development of a laboratory UWP GPR system for minedetection, Proc Hudem Symposium, RMA, Brussels, Belgium. 


\section{Figure legends}

Figure 1 The Tridem with the Metal Detector

Figure 2 The pneumatic walking robot AMRU 4

Figure 3 The Hunter with a blue beacon

Figure 4 The tracking system principle

Figure 5 The general system architecture

Figure 6a, $6 \mathrm{~b}$ and $6 \mathrm{c}$ : Translate mode, Spin mode and Free mode

Figure 7 Colour target identification

Figure 8 The CoRoDe data visualisation window

Figure 9 Data acquisition using the prototype GPR and a metal detector

Figures files are labelled "figX.ext"

\begin{tabular}{|l|l|}
\hline Files name & Mag factor \\
\hline Fig1.jpg & 100 \\
\hline Fig2.jpg & 100 \\
\hline Fig3.jpg & 100 \\
\hline Fig4.tif & 100 \\
\hline Fig5.tif & 80 \\
\hline Fig6.tif & 80 \\
\hline Fig7.tif & 100 \\
\hline Fig8.jpg & 70 \\
\hline Fig9.tif & 70 \\
\hline
\end{tabular}




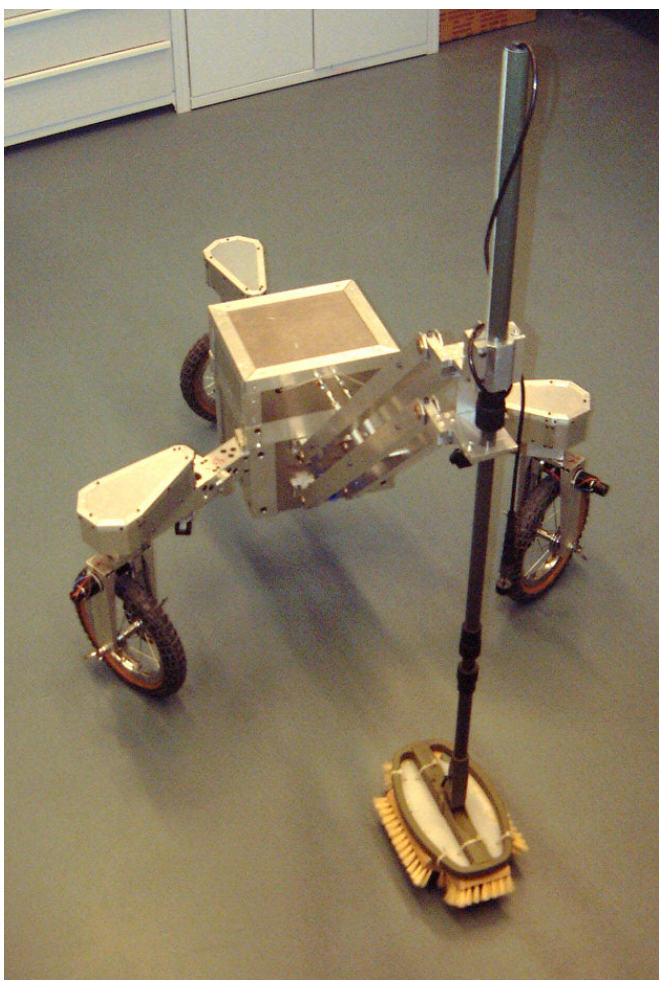

Figure 1. The Tridem with the Metal Detector

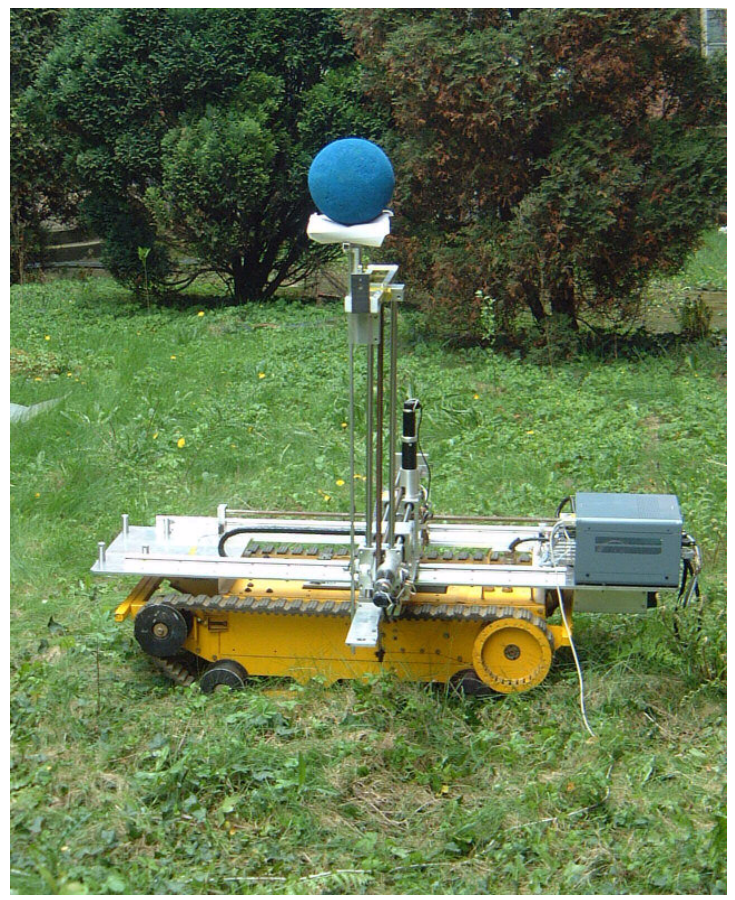

Figure 3 The Hunter with a blue beacon

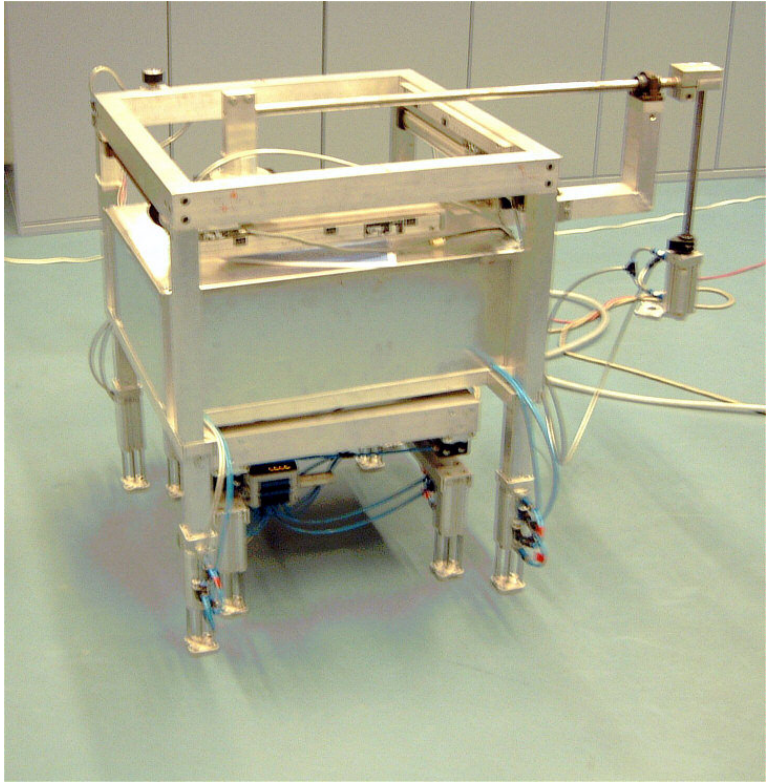

Figure 2. The pneumatic walking robot AMRU 4

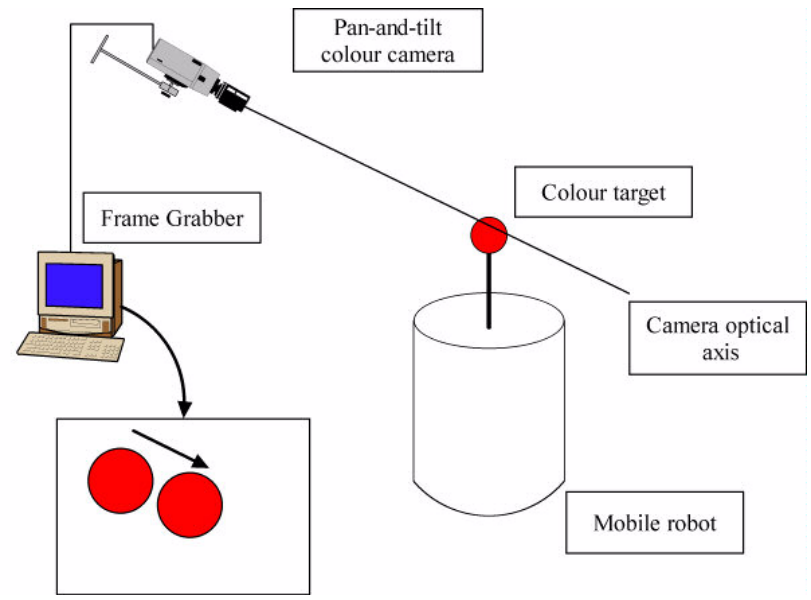

Figure 4 The tracking system principle 


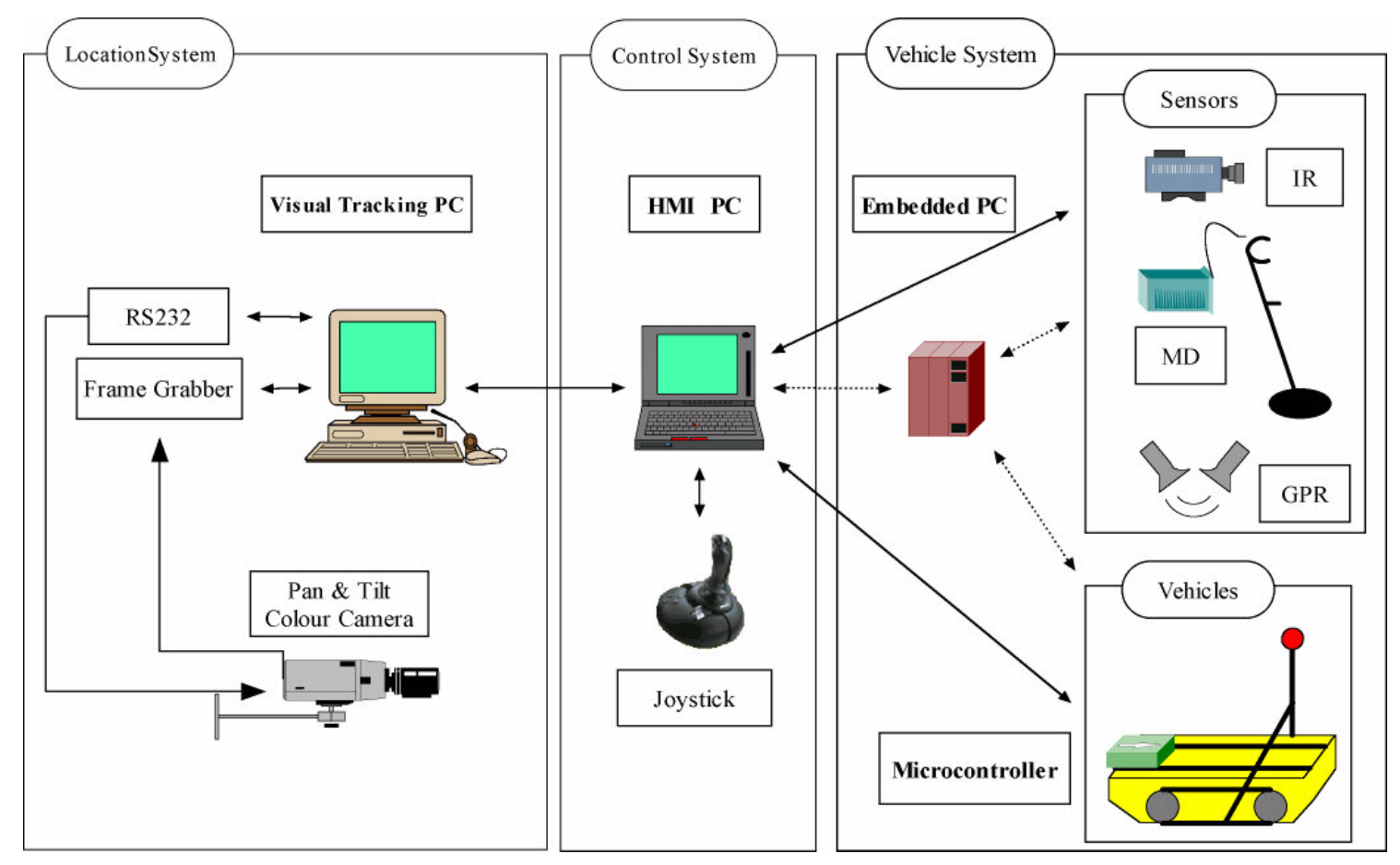

Figure 5 The general system architecture

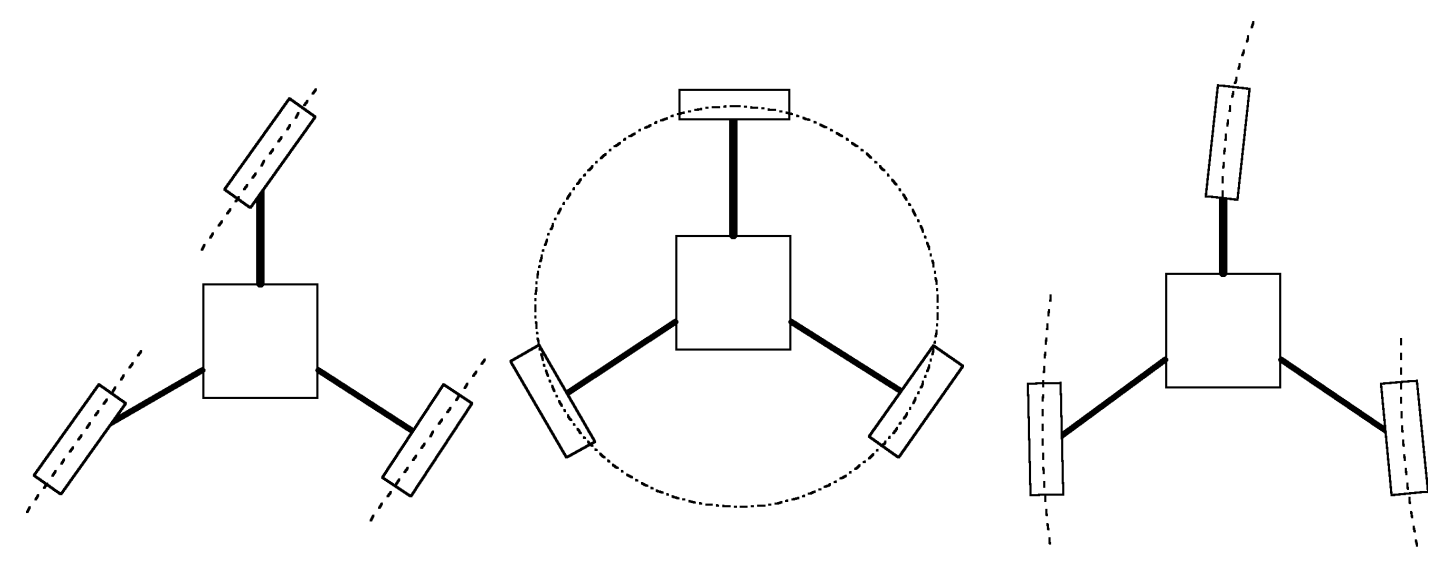

Figure 6a Translate mode

Figure $6 \mathrm{~b}$ Spin mode

Figure 6c Free mode 


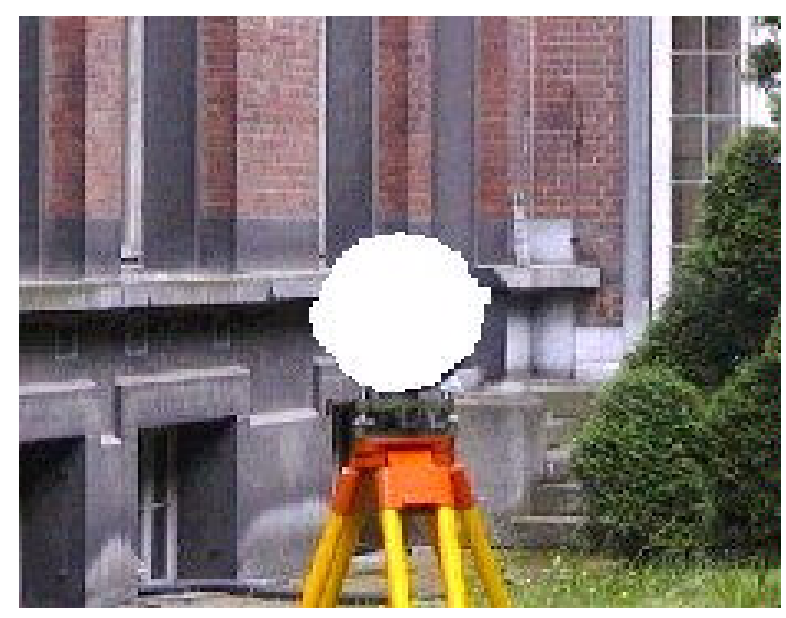

Figure 7 Colour target identification

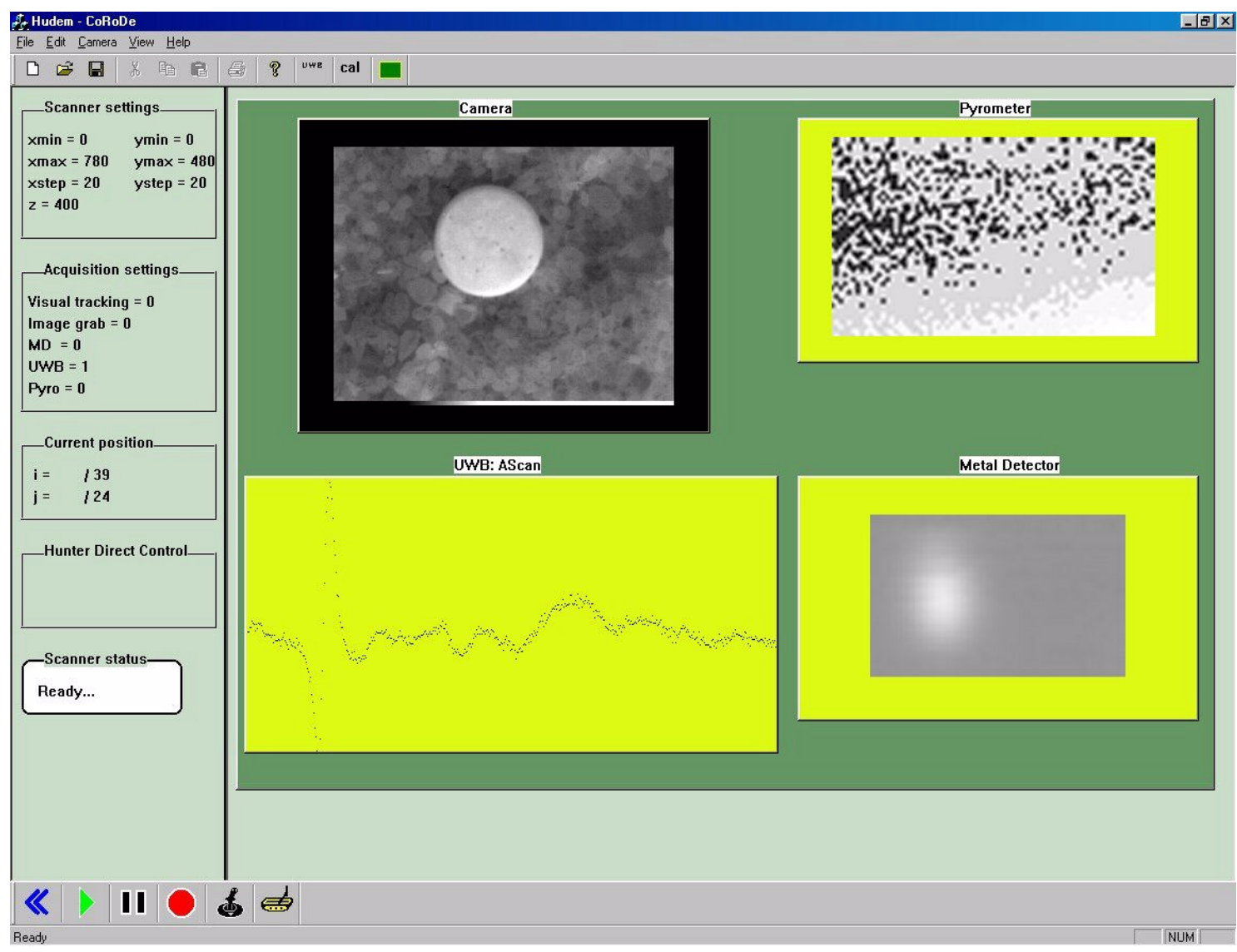

Figure 8 The CoRoDe data visualisation window 


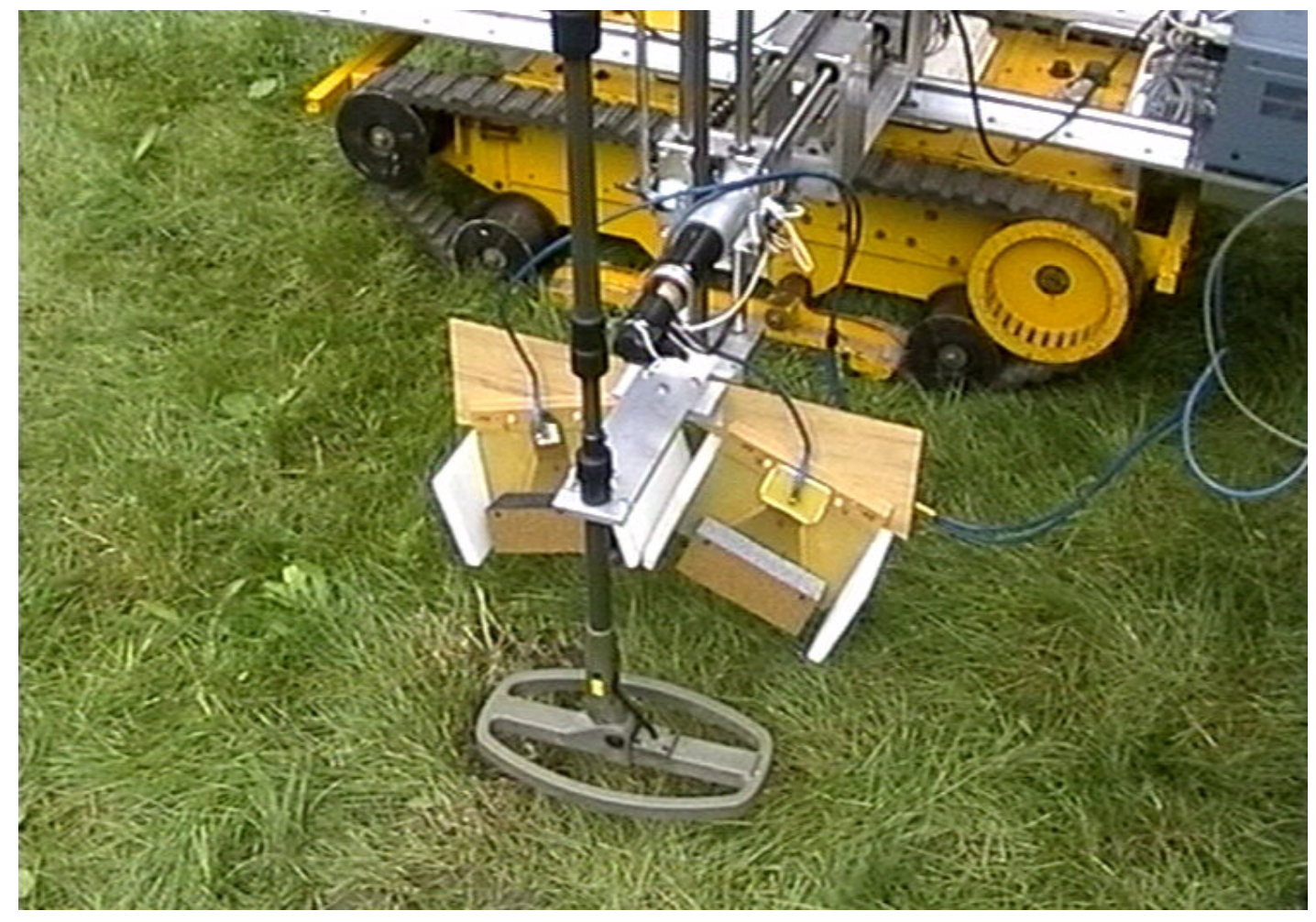

Figure 9 Data acquisition using the prototype GPR and a metal detector 